Case Report

\title{
Elevated Testosterone and Progestin Concentrations in a Spayed Female Rabbit with an Adrenal Cortical Adenoma
}

\author{
Katherine Baine, ${ }^{1}$ Kim Newkirk, ${ }^{2}$ Kellie A. Fecteau, ${ }^{2}$ and Marcy J. Souza ${ }^{2}$ \\ ${ }^{1}$ Department of Small Animal Clinical Sciences, College of Veterinary Medicine, University of Tennessee, 2407 River Drive, \\ Knoxville, TN 37996, USA \\ ${ }^{2}$ Department of Biomedical and Diagnostic Sciences, College of Veterinary Medicine, University of Tennessee, 2407 River Drive, \\ Knoxville, TN 37996, USA \\ Correspondence should be addressed to Katherine Baine; tnexoticpetvet@gmail.com
}

Received 22 July 2014; Accepted 24 November 2014; Published 8 December 2014

Academic Editor: Maria Teresa Mandara

Copyright (๑) 2014 Katherine Baine et al. This is an open access article distributed under the Creative Commons Attribution License, which permits unrestricted use, distribution, and reproduction in any medium, provided the original work is properly cited.

This case was described briefly in a recent book chapter (Lennox AM, Fecteau KA: 2014, Endocrine disease. In: BSAVA Manual of Rabbit Medicine, eds. Meredith A, Lord B, pp 274-276. British Small Animal Veterinary Association, Gloucester, UK). In the previous description, the tumor was described as a pheochromocytoma; however, further evaluation suggested that it more closely resembled an adrenal cortical adenoma. A 10-year-old, spayed female rabbit was presented for a behavior change of 8 months' duration. The rabbit was inappropriately urinating and defecating, as well as demonstrating aggressive behaviors such as chasing, biting, and mounting various objects. The rabbit had elevated progesterone, 17-hydroxyprogesterone, and testosterone concentrations, and ultrasound examination of the abdomen showed a round, homogenous nodule measuring $1.1 \times 0.8 \times 0.9 \mathrm{~cm}$ in the region of the left adrenal gland. Necropsy revealed a unilateral adrenal cortical adenoma. To the authors' knowledge, this is the first complete description of a female rabbit with an adrenal cortical adenoma documented in the literature.

\section{Case Presentation}

A 10-year-old, spayed female rabbit was presented for a behavior change of 8 months' duration. The rabbit was inappropriately urinating and defecating, as well as demonstrating aggressive behaviors such as chasing, biting, and mounting various objects. The rabbit had been spayed in the first year of life.

On physical examination, the only significant finding was an enlarged clitoris (Figure 1). No significant abnormalities were present on either a complete blood count or biochemistry panel. However, the rabbit had elevated blood levels of progesterone $(0.63 \mathrm{ng} / \mathrm{mL}$; reference interval for a spayed female rabbit (RISF) $0.09-0.47 \mathrm{ng} / \mathrm{mL}$ ), $17-\mathrm{OH}$ progesterone $(>25.0 \mathrm{ng} / \mathrm{mL}$, extrapolated result $60.2 \mathrm{ng} / \mathrm{mL}$; RISF $0.7-18.9 \mathrm{ng} / \mathrm{mL})$, and testosterone $(1.02 \mathrm{ng} / \mathrm{mL}$; RISF $0.02-$ $0.03 \mathrm{ng} / \mathrm{mL}$ ) [1]. Cortisol $(6.2 \mathrm{ng} / \mathrm{mL}$; RISF $4.9-10.1 \mathrm{ng} / \mathrm{mL})$ and androstenedione ( $1.2 \mathrm{ng} / \mathrm{mL}$; RISF $0.96-4.0 \mathrm{ng} / \mathrm{mL}$ ) levels were within normal limits [1]. Cortisol, progesterone, and testosterone assays (Coat-a-Count) were from Siemens
Medical Solutions Diagnostics, Los Angeles, CA, and 17OHP and androstenedione assays (ImmunChem Double Antibody) were from MP Biomedicals, Solon, $\mathrm{OH}$.

Ultrasound examination of the abdomen showed a round, homogenous nodule measuring $1.1 \times 0.8 \mathrm{~cm}$ in the region of the left adrenal gland (normal adrenal gland $0.72 \times 0.37 \mathrm{~cm}$ ) [2]; the nodule had an echogenicity approximately equal to the renal cortex. The right adrenal gland was not identified. The remainder of the examination was unremarkable. Due to the combination of clinical signs, elevated sex hormones, and a potential adrenal gland nodule, adrenal cortical disease was suspected. The owner declined exploratory surgery of the abdomen and adrenalectomy; therefore, medical management was recommended to potentially help alleviate clinical signs. Medical management was declined as well.

Six months following diagnosis, the patient was presented for progressive difficulty ambulating and rear limb ataxia; euthanasia was elected. On necropsy, the clitoris was markedly enlarged (measurements not obtained). The right adrenal gland measured $0.9 \mathrm{~cm} \times 0.6 \mathrm{~cm}$ and had a $1: 1: 1$ 


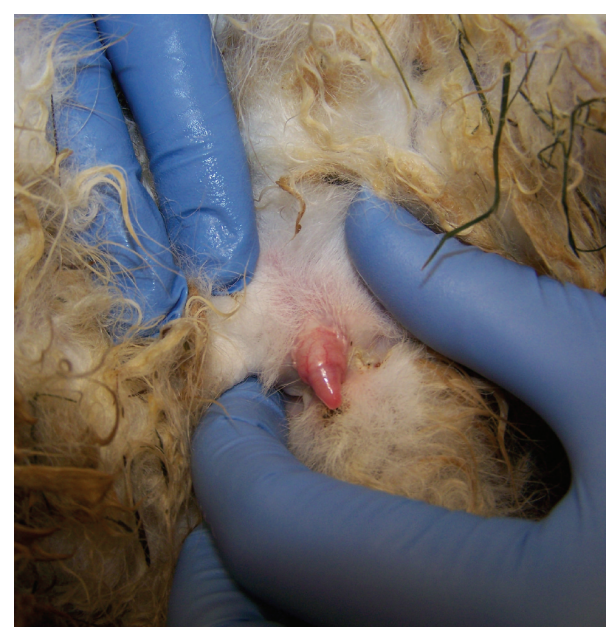

Figure 1: Clitoral enlargement in a 10 -year-old, spayed female rabbit.

ratio of thickness of cortical layers and medulla. The left adrenal measured $1 \mathrm{~cm} \times 1 \mathrm{~cm}$. On cut section, the cortex was enlarged by a tan to red mass that compressed and replaced the medulla. The left adrenal gland was closely associated with the left renal vein. The kidneys were dark brown with many $<1$-mm depressions within the capsule; on cut section was white streaking within the right renal pelvis. Samples were collected and fixed in 10\% neutral-buffered formalin and routinely processed for microscopic examination. Sections from each sample were stained with hematoxylin and eosin for further evaluation.

Histopathology revealed a neoplasm composed of cords and sheets of round to polygonal cells that expanded the left adrenal gland, compressed the cortex, and replaced the medulla (Figure 2(a)). The cells were frequently separated by vascular spaces and had distinct borders and moderate amounts of eosinophilic, often vacuolated cytoplasm. Nuclei were round and often had a single prominent nucleolus. There was minimal anisocytosis and anisokaryosis, and mitoses were rare to absent (Figure 2(a)). Only a small area of remaining medulla was present. A Churukian-Schenk stain, which highlights argyrophilic neurosecretory granules in the adrenal medulla, did not identify any granules in the neoplastic cells; however, a small piece of remaining adrenal medulla was positive (Figures 2(c) and 2(d)).

There was bilateral extracapsular adrenal cortical hyperplasia (Figure 2(a)). Both kidneys had multifocal linear areas where increased numbers of lymphocytes and plasma cells were present within the interstitium. The surrounding tubules were often atrophied and had a flattened epithelium (restitution) or hypertrophic cells (regeneration). Rare epithelial cells in these areas contained gram-positive organisms that were $2 \mu \mathrm{m}$ in diameter (suspected Encephalitozoon cuniculi). In the brain were multifocal areas in the meninges where blood vessels were surrounded by small numbers of lymphocytes and plasma cells. These areas extended into the VirchowRobin spaces of the underlying neuropil, with rare foci of lymphocytes within the neuropil. Frequently, neurons contained lipofuscin; scattered vessels were also surrounded by lipofuscin-laden macrophages. Similar findings were present in multiple sections of the spinal cord. In addition, there were areas where scattered axons had dilated myelin sheaths, with rare macrophages within the myelin sheaths and occasional swollen axons. No infectious agents were identified within the brain and spinal cord with gram staining.

The final diagnoses included left adrenal cortical adenoma, widespread multifocal lymphocytic interstitial nephritis with intralesional protozoa (suspected E. cuniculi), multifocal mild lymphocytic meningoencephalitis, widespread multifocal mild lymphocytic neuritis, and myelitis with Wallerian degeneration (suspected E. cuniculi associated).

\section{Discussion}

Adrenocortical disease associated with elevated sex hormones has been diagnosed in many species, including canines, felines, ferrets, and humans [3-9]. In veterinary medicine, adrenocortical disease associated with elevated sex hormones has also been infrequently reported in rabbits: in 5 male and 1 additional female rabbit $[5,6,9]$. The pathogenesis of disease in rabbits is not well understood, but some hypothesize a similar mechanism to ferrets and mice in which gonadectomy contributes to adrenocortical hyperplasia and later tumorigenesis [3, 4, 10-12]. It is suspected that adrenocortical hyperplasia is secondary to the loss of feedback inhibition from the gonads to the hypothalamicpituitary axis, which leads to chronic elevation in circulating luteinizing hormone, causing synthesis and secretion of sex steroids from the adrenal cortex $[4,12]$. A previous study has suggested adrenal sex hormone production in normal ovariectomized rabbits, further supporting this hypothesis. Juvenile female rabbits after ovariectomy demonstrated continued sexual behaviors, which were then significantly reduced after adrenalectomy [13]. In contrast, ovariectomy eliminated all sexual behaviors in rats [14]. Given the increasing number of reported cases of adrenocortical disease in rabbits, additional research is needed to elucidate the pathophysiology of the disease in rabbits.

Based on other reported cases of adrenal cortical disease in rabbits, male rabbits are overrepresented 5 to $1[5,6,9]$, and the average age of onset of clinical signs is reported to be 7.6 years old [5]. The age of onset of clinical signs in this case was 10 years. Except for the clitoral enlargement, the clinical signs in this case were similar to previous descriptions of rabbits with adrenal cortical disease, including urine and/or fecal marking, aggressive behaviors, and mounting various objects.

The diagnostic workup in this case was highly suggestive for adrenocortical disease; however, the right adrenal gland was not identified on ultrasound, and the left adrenal gland was not definitively identified and measured. Given the size, location, and appearance of the identified nodule, an association with the left adrenal gland was suspected.

Sex hormone reference ranges have been established for neutered rabbits of both sexes [1]. Unlike other described rabbit cases with adrenal cortical disease, including the other 


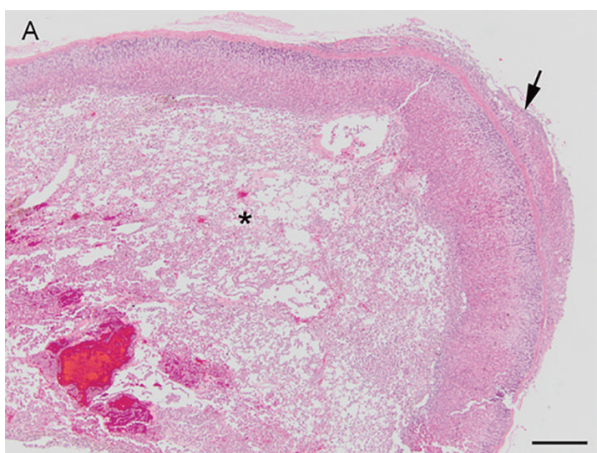

(a)

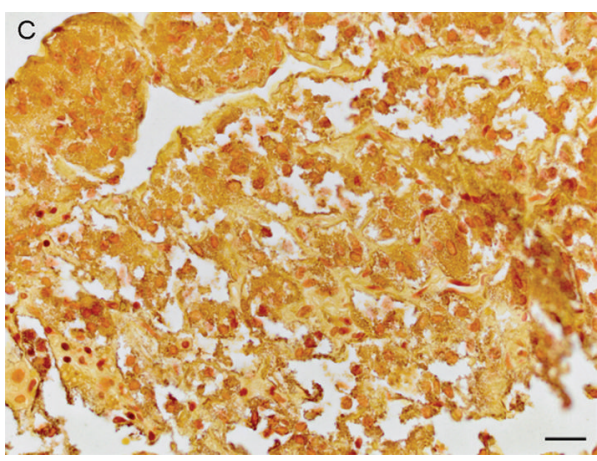

(c)

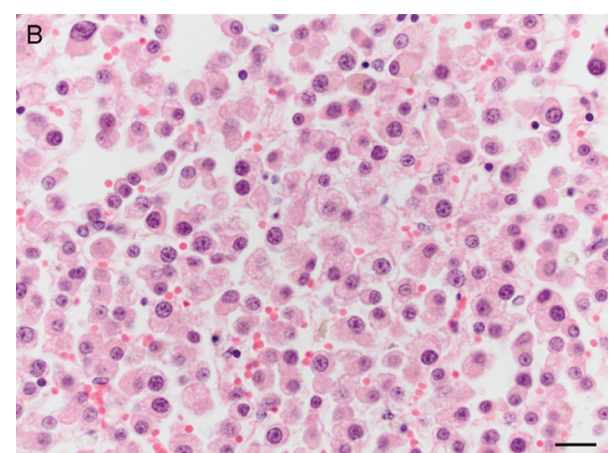

(b)

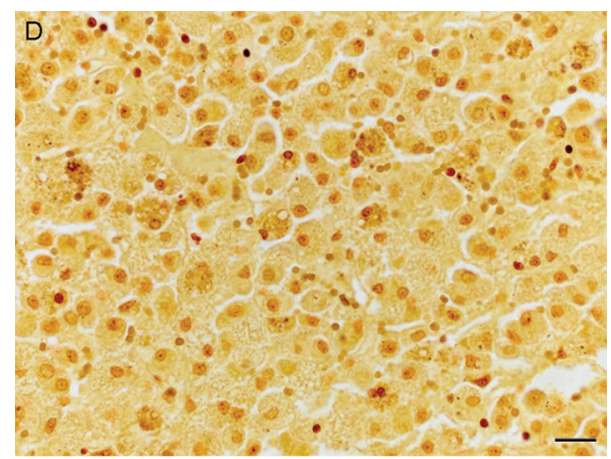

(d)

FIgURE 2: Adrenal gland from a rabbit. (a) The adrenal medulla is replaced by a well-demarcated mass of neoplastic cells (asterisk), which compresses the overlying adrenal cortex. There is also extracapsular adrenal cortical hyperplasia (arrow). Hematoxylin and eosin (H\&E) stain. Bar $=200 \mu \mathrm{m}$. (b) The neoplastic cells are round to polygonal and occasionally form cords or nests, which are separated by vascular spaces. Occasionally the cytoplasm is vacuolated. $\mathrm{H} \& \mathrm{E}$ stain. Bar $=10 \mu \mathrm{m}$. (c) Compressed normal adrenal medulla with brown cytoplasmic granules. Churukian-Schenk stain. Bar $=10 \mu \mathrm{m}$. (d) The neoplastic cells do not contain brown cytoplasmic granules. Churukian-Schenk stain. Bar $=$ $10 \mu \mathrm{m}$.

female rabbit mentioned briefly in a recent book chapter [5], progesterone and 17-hydroxyprogesterone concentrations were elevated in this case. Progesterone has been shown to induce a significant increase in aggression when administered to ovariectomized rabbits [15]. The increased levels in this case likely contributed to the aggression documented by the owner. Markedly elevated testosterone concentrations in this case likely also contributed to the clitoral enlargement and aggressive behaviors, which was similar to cases described with adrenal disease (aggressive behaviors) $[5,6$, 9]. Testosterone levels were consistently increased in other cases of adrenocortical disease in rabbits, possibly due to the predominance of male rabbits previously described [5]. Additional possible causes for elevated sex hormones in a castrated animal include gonadal remnant, ectopic gonadal tissue, or other neoplasia. Human chorionic gonadotropin (hCG) stimulation testing has been used in rabbit patients to determine if an ovarian remnant or ectopic gonadal tissue is present $[1,15]$; however, such testing was not pursued in this case.

Ultimately, the diagnosis of a unilateral adrenocortical adenoma was made on necropsy. Although extracapsular hyperplasia was identified bilaterally, it is an incidental finding in older horses, cats, and dogs [16]. Previous reports of adrenal cortical disease in rabbits describe unilateral hyperplasia and carcinoma; adenomas have not been reported $[5,6,9]$. The adenoma was likely the cause for the elevated progesterone and testosterone in this case; however, since treatment was not pursued antemortem and immunohistochemical staining was not performed postmortem, there was no direct evidence that the adenoma caused increased hormone levels. A decreased testosterone level has been demonstrated in other cases of adrenal disease in rabbits after complete adrenalectomy [5].

The owner declined treatment for suspected adrenocortical disease in this case. The treatment of choice for unilateral disease is adrenalectomy [5], and although surgical removal may have been challenging due to the close association of the tumor to the left renal vein, complete surgical removal likely would have been curative. Medical management of adrenocortical disease using deslorelin, leuprolide acetate, finasteride, trilostane, or flutamide has been attempted in rabbits with variable success [5]. More research needs to be performed to evaluate the efficacy and safety of these therapies in rabbits.

Despite independent of the adrenal disease, ultimately, the clinical signs for which the patient was euthanized were secondary to E. cuniculi lesions within the central nervous 
system and kidneys. Encephalitozoonosis is a common disease of rabbits caused by obligate intracellular microsporidia. It can cause a nonsuppurative to granulomatous encephalitis and chronic interstitial nephritis [17]. Patients therefore can show a variety of neurologic signs and/or kidney failure. The rabbit in this case showed signs of neurologic disease prior to euthanasia (difficulty ambulating, ataxia); however, no clinical signs associated with renal disease were noted (polyuria/polydipsia) despite lesions found within the kidneys. Antemortem diagnostics were not performed to identify the underlying cause for the neurologic signs or determine if renal failure was present, although blood work performed 6 months previously was within normal limits. The lesions identified in the central nervous system and kidneys on histopathology were consistent with E. cuniculi; however, immunohistochemistry or polymerase chain reaction is required for a definitive diagnosis [17].

This case highlights the clinical signs, diagnostic workup, and necropsy findings associated with a unilateral adrenal cortical adenoma in an older, spayed female rabbit. Adrenal cortical disease should be considered in a spayed female rabbit demonstrating sexual behaviors (aggression, marking behaviors) and/or with an enlarged clitoris.

\section{Conflict of Interests}

The authors declared no potential conflict of interests with respect to the research, authorship, and/or publication of this paper.

\section{Acknowledgment}

The authors would like to thank Misty Bailey for editorial support.

\section{References}

[1] K. A. Fecteau, B. J. Deeb, J. M. Rickel, W. J. Kelch, and J. W. Oliver, "Diagnostic endocrinology: blood steroid concentrations in neutered male and female rabbits," Journal of Exotic Pet Medicine, vol. 16, no. 4, pp. 256-259, 2007.

[2] S. Reese, "Abdomen," in Diagnostic Imaging of Exotic Pets, M. E. Krautwald-Junghanns, M. Pees, S. Reese, and T. Tully, Eds., p. 236, Schlütersche Verlagsgesellschaft mbH \& Co. KG, Hannover, Germany, 2011.

[3] F. Beuschlein, S. Galac, and D. B. Wilson, "Animal models of adrenocortical tumorigenesis," Molecular and Cellular Endocrinology, vol. 351, no. 1, pp. 78-86, 2012.

[4] M. Bielinska, S. Kiiveri, H. Parviainen, S. Mannisto, M. Heikinheimo, and D. B. Wilson, "Gonadectomy-induced adrenocortical neoplasia in the domestic ferret (Mustela putorius furo) and laboratory mouse," Veterinary Pathology, vol. 43, no. 2, pp. 97117, 2006.

[5] A. M. Lennox, "Surgical treatment of adrenocortical disease," in BSAVA Manual of Rabbit Surgery, Dentistry, and Imaging, F. Harcourt-Brown and J. Chitty, Eds., pp. 269-272, British Small Animal Veterinary Association, Gloucester, UK, 2014.

[6] A. M. Lennox and J. Chitty, "Adrenal neoplasia and hyperplasia as a cause of hypertestosteronism in two rabbits," Journal of Exotic Pet Medicine, vol. 15, no. 1, pp. 56-58, 2006.
[7] E. N. Meler, J. C. Scott-Moncrieff, A. T. Peter et al., "Cyclic estrous-like behavior in a spayed cat associated with excessive sex-hormone production by an adrenocortical carcinoma," Journal of Feline Medicine and Surgery, vol. 13, no. 6, pp. 473$478,2011$.

[8] H. M. Syme, J. C. Scott-Moncrieff, N. G. Treadwell et al., "Hyperadrenocorticism associated with excessive sex hormone production by an adrenocortical tumor in two dogs," Journal of the American Veterinary Medical Association, vol. 219, no. 12, pp. $1725-1728,2001$.

[9] M. Varga, "Hypersexuality in a castrated rabbit (Oryctolagus cuniculus)," Companion Animal, vol. 16, no. 1, pp. 48-51, 2011.

[10] M. K. de Jong, E. E. M. ten Asbroek, A. J. Sleiderink, A. J. Conley, J. A. Mol, and N. J. Schoemaker, "Gonadectomy-related adrenocortical tumors in ferrets demonstrate increased expression of androgen and estrogen synthesizing enzymes together with high inhibin expression," Domestic Animal Endocrinology, vol. 48, no. 1, pp. 42-47, 2014.

[11] N. J. Shoemaker, M. Schuurmans, H. Moorman, and J. T. Lumeij, "Correlation between age at neutering and age at onset of hyperadrenocorticism in ferrets," Journal of the American Veterinary Medical Association, vol. 216, no. 2, pp. 195-197, 2000.

[12] N. J. Schoemaker, K. J. Teerds, J. A. Mol, J. T. Lumeij, J. H. H. Thijssen, and A. Rijnberk, "The role of luteinizing hormone in the pathogenesis of hyperadrenocorticism in neutered ferrets," Molecular and Cellular Endocrinology, vol. 197, no. 1-2, pp. 117125, 2002.

[13] C. Beyer, M. L. Cruz, and N. Rivaud, "Persistence of sexual behavior in ovariectomized-adrenalectomized rabbits treated with cortisol," Endocrinology, vol. 85, no. 4, pp. 790-793, 1969.

[14] C. Beyer, K. L. Hoffman, and O. González-Flores, "Neuroendocrine regulation of estrous behavior in the rabbit: similarities and differences with the rat," Hormones and Behavior, vol. 52, no. 1, pp. 2-11, 2007.

[15] K. L. Hoffman, E. Martínez-Alvarez, and R. I. Rueda-Morales, "The inhibition of female rabbit sexual behavior by progesterone: progesterone receptor-dependent and-independent effects," Hormones and Behavior, vol. 55, no. 1, pp. 84-92, 2009.

[16] C. C. Capen, "Endocrine system," in Jubb, Kennedy and Palmers Pathology of Domestic Animals, M. G. Maxie, Ed., pp. 325-482, Elsevier, Toronto, Canada, 5th edition, 2007.

[17] M. Leipig, K. Matiasek, H. Rinder et al., "Value of histopathology, immunohistochemistry, and real-time polymerase chain reaction in the confirmatory diagnosis of Encephalitozoon cuniculi infection in rabbits," Journal of Veterinary Diagnostic Investigation, vol. 25, no. 1, pp. 16-26, 2013. 

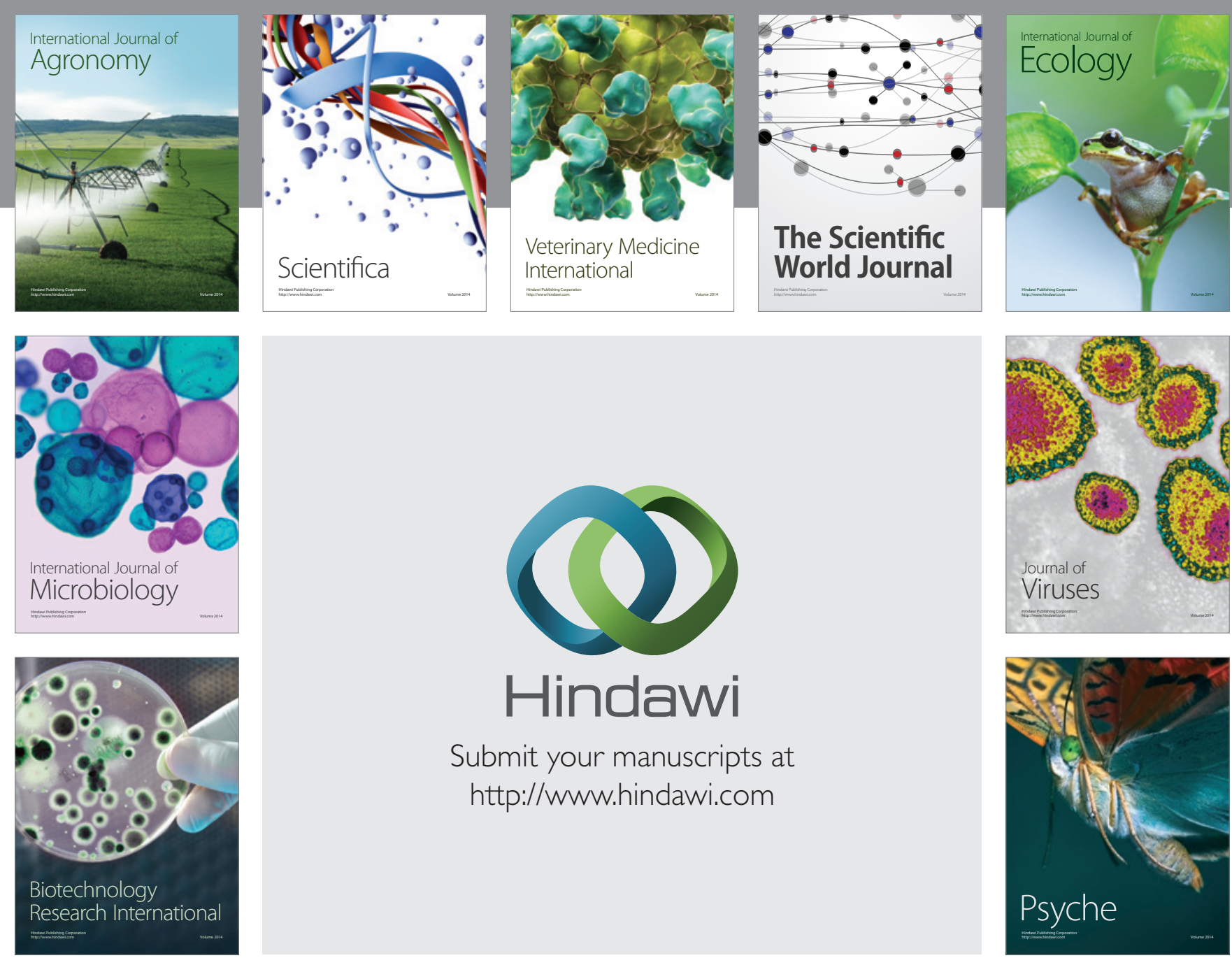

Submit your manuscripts at http://www.hindawi.com
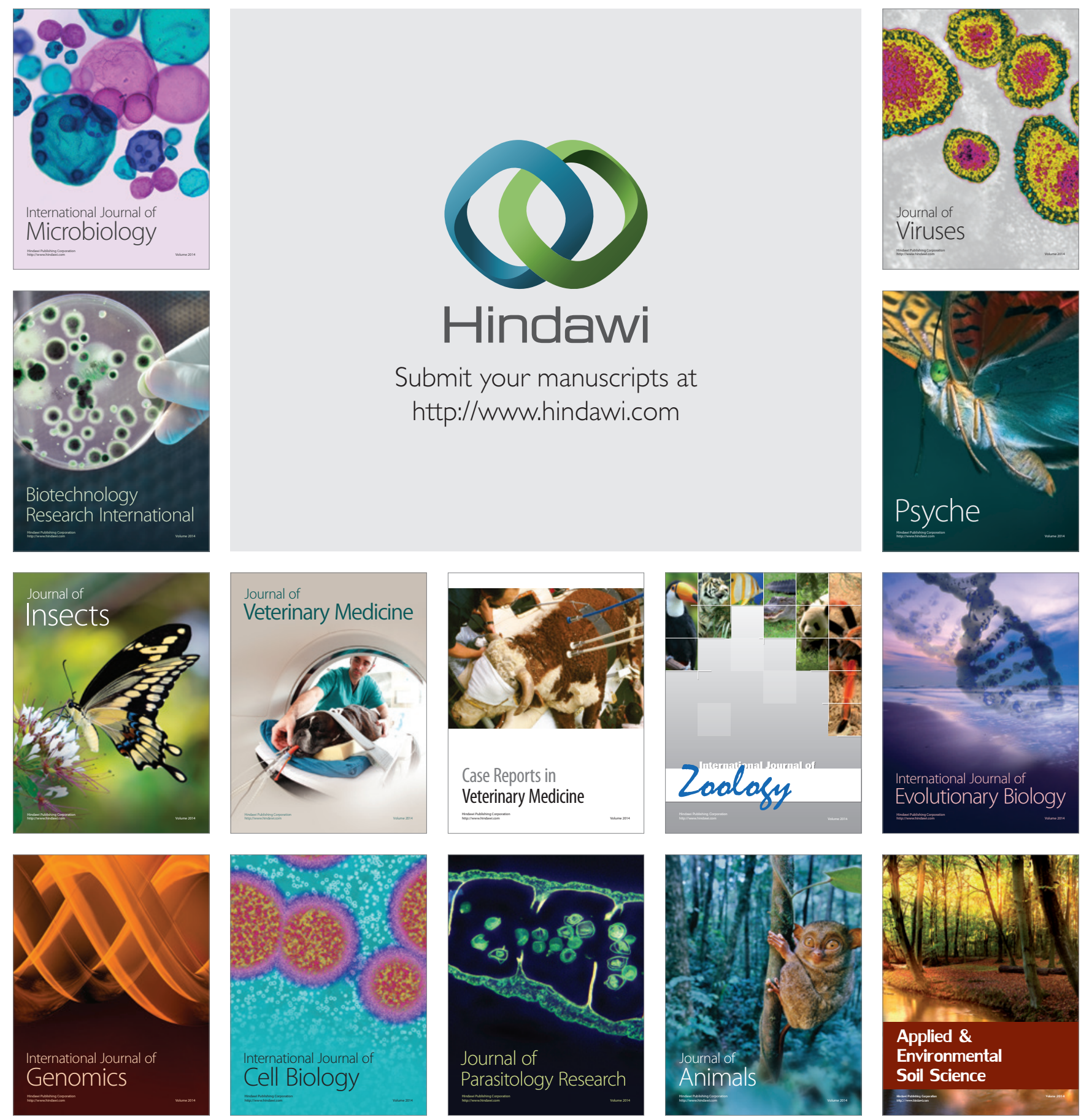